\title{
Detection of Vertical Root Fractures Using Three Different Imaging Modalities: An In Vitro Study
}

\author{
Dunia Al Hadi ${ }^{1}$, Sana Parekh² ${ }^{2}$ Warda Naeem ${ }^{3}$, Alexander M Luke ${ }^{4}$, Simy Mathew ${ }^{5}$
}

\begin{abstract}
Background: The diagnosis of nondisplaced longitudinal fractures [vertical root fractures (VRFs)] is challenging in clinical practice. Radiographic techniques showed a difficulty in detection of VRFs. Cone beam computed tomography (CBCT) is a new diagnostic imaging modality that provides high-quality three-dimensional (3D) images for dental diagnosis.

Aims: The aim of this in vitro study is to compare accuracy of three different imaging modalities: conventional periapical radiographs, digital radiographs, and CBCT in detecting VRFs in teeth that are endodontically as well as non-endodontically treated.

Materials and methods: An in vitro model consisting of 60 recently extracted human mandibular lower premolars were used. Root canal treatment was carried out for 30 teeth. Root fractures were created in 30 teeth ( 15 root canal treated and 15 non-treated) by mechanical force. Other 30 teeth remain intact. The teeth were mounted and images were taken with a periapical, digital, and CBCTX-ray unit. Three endodontists separately evaluated the images.

Results: Interobserver $\kappa$ values showed a very good interobserver agreement $(0.98$ for CBCT, 0.88 for digital, and 0.93 for conventional periapical $\mathrm{X}$-rays). There was an overall statistically significant difference $(p=0.00)$ in detecting of root fracture among the three imaging modalities and the highest accuracy with CBCT images.

Conclusions: In in vitro model, CBCT scan appears to give the highest accuracy in detecting VRFs when compared with the periapical systems in both endodontically and non-endodontically treated teeth.

Clinical significance: The CBCT scan shows higher sensitivity in detection of VRFs in comparison with periapical images.

Keywords: Cone beam computed tomography, Laboratory research, Root canal treatment, Vertical root fractures.

The Journal of Contemporary Dental Practice (2020): 10.5005/jp-journals-10024-2839
\end{abstract}

\section{INTRODUCTION}

Vertical root fracture (VRF) is defined as a complete or incomplete fracture initiated from the root at any level, usually directed bucco-lingually. ${ }^{1}$ According to the literature, VRF is the third most common reason for extraction of an endodontically treated tooth. ${ }^{2}$ It has been suggested that improper selection of intracanal posts and cementation techniques or excessive pressure during lateral condensation of gutta-percha are among the main etiological factors causing root fractures. ${ }^{3}$

Testori et al. ${ }^{4}$ reported that premolars have the highest incidence of VRF in endodontically treated teeth; however, Chan et al. ${ }^{5}$ reported first molars to be the teeth with higher frequency of root fractures.

Vertical root fractures are also prevalent in vital teeth (nonendodontically treated teeth). In vital teeth, VRFs occur due to factors such as repetitive and heavy masticatory stress referred to as "fatigue root fractures," ${ }^{\text {"6 }}$ strong masticatory forces, habitual chewing of hard food, and less pliable supporting bone. ${ }^{7}$

The diagnosis of VRFs is a significant challenge for the dental practitioner. ${ }^{3,8}$ Vertical root fractures are difficult to diagnose using conventional and digital radiographic methods, and the line of fracture could often be overlooked if the $X$-ray beam does not pass parallel through it. ${ }^{9}$ Hence, at least two periapical radiographs, with different angulations, are needed in order to detect such fractures.

Digital radiography uses sensors instead of traditional photographic film and special image processing techniques that enhance overall display of the image and the quality can be enhanced and zoomed in and out. However, studies have shown that digital radiography provides equivalent results when
${ }^{1}$ Department of Restorative Dentistry, College of Dentistry, Ajman University, Ajman, United Arab Emirates

${ }^{2,3}$ College of Dentistry, Ajman University, Ajman, United Arab Emirates ${ }^{4}$ Department of Surgical Sciences, College of Dentistry, Ajman University, Ajman, United Arab Emirates

${ }^{5}$ Department of Growth and Development, College of Dentistry, Ajman University, Ajman, United Arab Emirates

Corresponding Author: Alexander M Luke, Department of Surgical Sciences, College of Dentistry, Ajman University, Ajman, United Arab Emirates, Phone: +971 555171094, e-mail: alexvinod@gmail.com

How to cite this article: Hadi DA, Parekh S, Naeem W, et al. Detection of Vertical Root Fractures Using Three Different Imaging Modalities: An In Vitro Study. J Contemp Dent Pract 2020;21(5):549-553.

Source of support: Nil

Conflict of interest: None

compared with an F-speed conventional radiographic film in the detection of VRFs in single-rooted teeth. ${ }^{1}$ Cone beam computed tomography $(\mathrm{CBCT})$ is a diagnostic imaging modality that provides high-quality, accurate 3D images of the osseous elements of the maxillofacial skeleton. ${ }^{10}$

Previous studies have evaluated the accuracy of the CBCT system compared with digital and periapical radiographs in the detection of VRFs. While it is agreed that detection of VRFs is a challenge using two-dimensional (2D) radiographic images, there is no agreement on the accuracy of $C B C T$ imaging in detecting VRFs. ${ }^{11,12}$

() The Author(s). 2020 Open Access This article is distributed under the terms of the Creative Commons Attribution 4.0 International License (https://creativecommons. org/licenses/by-nc/4.0/), which permits unrestricted use, distribution, and non-commercial reproduction in any medium, provided you give appropriate credit to the original author(s) and the source, provide a link to the Creative Commons license, and indicate if changes were made. The Creative Commons Public Domain Dedication waiver (http://creativecommons.org/publicdomain/zero/1.0/) applies to the data made available in this article, unless otherwise stated. 
The aim of this in vitro study is to compare accuracy of three different imaging modalities: conventional periapical radiographs, digital radiographs, and $C B C T$ in detecting VRFs in teeth with and without root canal treatment.

\section{Materials and Methods}

The Research Ethics Committee of the Faculty of Dentistry (RD2014/15-01) approved the present study. In total, 100 extracted single-rooted lower premolars were collected for the study. The teeth were cleaned and disinfected and then examined under stereomicroscope, under the magnification of $8 \times$. These teeth were then mounted in wax, and preoperative periapical radiographs were taken to confirm teeth with single canals and absence of preexisting fractures, root resorptions, dental anomalies and confirm that they were not previously endodontically treated. The sample was placed in normal saline during all stages of the study.

After the preoperative assessment, 60 sound teeth were selected, and further divided into the following groups.

- Group I consisting of 30 teeth underwent root canal therapy;

- Group II consisting of 30 teeth received no root canal therapy.

Group I teeth were mounted in wax individually, and access cavities were prepared; the canals were then prepared using WaveOne reciprocating motor system and primer WaveOne file and obturated with the cold lateral compaction technique. Later, all teeth were decoronated to continue the experiment.

Group I was then subdivided into

Group 11: 15 teeth subjected to mechanical force using a hammer and nail to create a crack (nondisplaced VRF).

Group 12: 15 teeth not subjected to any mechanical force (no root fracture).

Group II subdivided into

Group II1: 15 teeth subjected to mechanical force using the Instron Universal Testing Machine to create a vertical crack (nondisplaced VRF).

Group II2: 15 intact teeth.

Teeth were reexamined under the stereomicroscope, under the magnification of $8 \times$ to detect the presence of the cracks (nondisplaced VRF) (Fig. 1).

Before radiographic examination, all teeth were mounted in wax and covered with putty material, so that it can replicate soft tissue and bone.

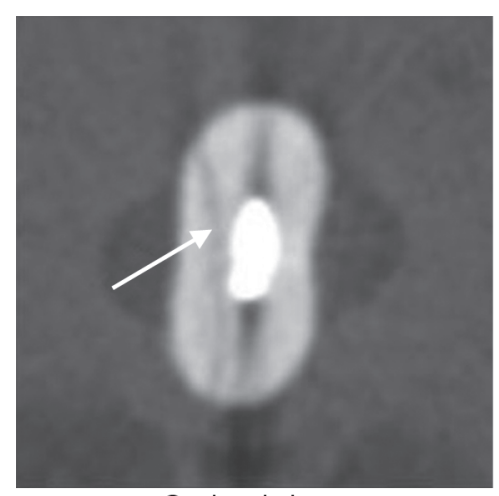

Occlusal view

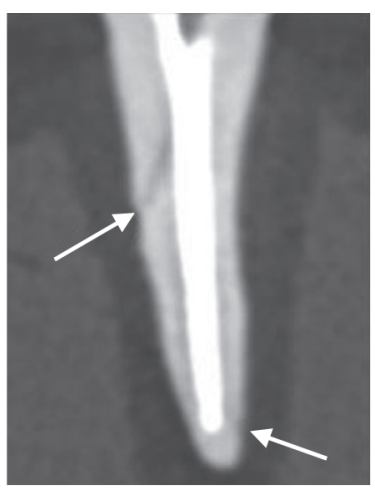

Oblique slice
Fig. 1: Vertical root fracture detected by cone beam computed tomography images

\section{Imaging Techniques}

All teeth were then subjected to the three different imaging modalities: conventional periapical, digital, and CBCT.

Cone beam computed tomography images were obtained using Carestream ${ }^{\circledast}$ CS 9000 3D CBCT using the following exposure factors: $60 \mathrm{kVp}, 5 \mathrm{~mA}$, and $10 \mathrm{~ms}$. Scans of the sample made with $3.7 \times 5 \mathrm{~cm}$ field of view selection and $76 \times 76 \times 76 \mu \mathrm{m}$ (isotropic voxel) was used (Fig. 1).

Images were analyzed through the Digital Imaging Software CS 3.7.5 for CBCT machine.

Digital intraoral radiograph was taken using Carestream 6200 at two different horizontal angles $\left(0^{\circ}\right.$ and $15^{\circ}$ mesial) using the following exposure factors: $60 \mathrm{kVp}, 7 \mathrm{~mA}$, and 0.20 seconds. Images were analyzed with Digital Imaging Software CS 3.7.5 for CBCT machine. Periapical images were taken using Carestream 2200 at two different horizontal angles $\left(0^{\circ}\right.$ and $15^{\circ}$ mesial) using the following exposure factors: $60 \mathrm{kVp}, 7 \mathrm{~mA}$, and 0.20 seconds. Periapical films were then processed in a standard pattern of developing, washing, fixing, and rewashing. The solutions were changed after every 10 periapical films were exposed.

\section{Image Assessment}

The images obtained were evaluated by three observers, two of whom were endodontists with more than 5 years of experience in clinical practice and one was a general dental practitioner. The criteria decided for the detection of VRFs was direct visualization of a radiolucent line on the root surface. The fracture/non-fracture assessment was recorded as a binary score: 1 if a fracture was present or 0 if it was not. The decisions of the observers were not influenced by any of the researchers. The images were displayed and analyzed on a computer monitor. Adjustment of contrast and brightness been applied, if considered necessary, using the inbuilt image processing tools.

\section{Statistical Analysis}

Data analysis was performed using IBM SPSS Version 20. $\kappa$ statistics was used to determine the interobserver reliability. Chi-square statistics was used to determine the differences in accuracy among the three imaging modalities. Results were considered significant at $p$ lower than 0.05 . Sensitivity and specificity for each imaging technique were calculated.

- Fracture/non-fracture scores were evaluated as follows: correct identification of a non-fractured root was considered as true negative (TN), correct identification of fracture site in a fractured root was identified as true positive (TP), identification of a fracture in a non-fractured root was identified as false-positive (FP), incorrect identification of a fracture site in a fractured root was identified as falsenegative (FN), and failure to identify a fracture in a fractured root was recorded as FN.

- Sensitivity, specificity, accuracy, positive predictive value, and negative predictive value among the three imaging modalities were calculated as follows:

Sensitivity $=T P / T P+F N$,

Specificity $=$ TN $/ T N+F P$,

Accuracy $=\mathrm{TP}+\mathrm{TN} / \mathrm{TP}+\mathrm{TN}+\mathrm{FP}+\mathrm{FN}$,

Positive predictive value $=\mathrm{TP} / \mathrm{TP}+\mathrm{FP}$,

Negative predictive value $=\mathrm{TN} / \mathrm{TN}+\mathrm{FN}$ 
Table 1: Frequencies of vertical root fractures as seen in the different radiographic images in non-obturated teeth

\begin{tabular}{|c|c|c|c|c|c|c|c|c|c|}
\hline & \multicolumn{3}{|c|}{ Periapical } & \multicolumn{3}{|c|}{ Digital } & \multicolumn{3}{|c|}{$C B C T$} \\
\hline & Fractured & Not fractured & Total & Fractured & Not fractured & Total & Fractured & Not fractured & Total \\
\hline Fractured & & & & 38 & 0 & 38 & 45 & 0 & 45 \\
\hline Not fractured & 45 & 45 & 90 & 7 & 45 & 52 & 0 & 45 & 45 \\
\hline Total & 45 & 45 & 90 & 45 & 45 & 90 & 45 & 45 & 90 \\
\hline
\end{tabular}

Table 2: Frequencies of vertical root fractures as seen in the different radiographic images in obturated teeth

\begin{tabular}{|c|c|c|c|c|c|c|c|c|c|}
\hline & \multicolumn{3}{|c|}{ Periapical } & \multicolumn{3}{|c|}{ Digital } & \multicolumn{3}{|c|}{$C B C T$} \\
\hline & Fractured & Not fractured & Total & Fractured & Not fractured & Total & Fractured & Not fractured & Total \\
\hline Fractured & 15 & 1 & 16 & 39 & 7 & 46 & 42 & 0 & 42 \\
\hline Not fractured & 30 & 44 & 74 & 6 & 38 & 44 & 3 & 45 & 48 \\
\hline Total & 45 & 45 & 90 & 45 & 45 & 90 & 45 & 45 & 90 \\
\hline
\end{tabular}

\section{RESULTS}

The frequencies of VRF in each type of radiograph for nonobturated and obturated teeth are described in Tables 1 and 2.

The sensitivity and specificity, respectively, of the VRF diagnosis in the assessment of non-obturated canals using:

- Periapical radiographs could not be determined as they were all identified as non-fractured;

- Digital radiographs were 84.4 and $100 \%(\kappa=0.844, p=0.00)$;

- $\mathrm{CBCT}$ images were 100 and $100 \%(\kappa=1.00, p=0.000)$.

The sensitivity and specificity, respectively, of the VRF diagnosis in the assessment of obturated canals using:

- Periapical radiographs were 33.3 and $97.8 \%(\kappa=0.311, p=0.000)$;

- Digital radiographs were 86.7 and $84.4 \%(\kappa=0.711, p=0.00)$;

- Cone beam computed tomography images were 93.3 and $100 \%$ $(\kappa=0.933, p=0.000)$.

The accuracy of the various radiographic modalities in detecting VRF in non-obturated vs obturated teeth are depicted in Table 3.

The receiver operating characteristic (ROC) curve is important to assess the accuracy of the various radiographic images in detection of VRF in both obturated and non-obturated teeth. When comparing the area under curve in the assessment of the non-obturated teeth radiographic images, the periapical shows an area of 0.5 [confidence interval $(\mathrm{Cl}): 0.380-0.620, p=1.0$ ], the digital radiographic image gives an area of $0.922(\mathrm{Cl}: 0.858-0.986$, $p=0.033$ ), and the CBCT imaging an area of $1.000(p=0.000)$ (Fig. 2).

In the assessment of VRF in the radiographs of the obturated teeth, the periapical radiographs show an area of 0.656 (Cl: $0.542-$ $0.770, p=0.11)$, the digital radiographic image gives an area of 0.856 (Cl: 0.771-0.940, $p=0.000$ ), and the CBCT imaging describes an area of 0.967 (Cl: 0.924-1.00, $p=0.000$ ) (Fig. 3).

\section{Discussion}

This study compared three radiographic systems, CBCT, digital, and conventional film-based periapical radiography, for their accuracy in detecting experimental root fractures. The statistical analysis showed that there is a significant difference in obtaining images of the fractured teeth between conventional periapical and digital or CBCT techniques.

In vitro simulating models are not realistic and hardly represent the clinical situations; however, in in vivo studies, the presence or
Table 3: Accuracy of different radiographic image modalities in detecting vertical root fractures in obturated and non-obturated teeth

\begin{tabular}{lll}
\hline & Non-obturated (\%) & Obturated (\%) \\
\hline $\begin{array}{l}\text { Conventional } \\
\text { periapical }\end{array}$ & - & 65.5 \\
Digital & 92.2 & \\
CBCT & 100 & 85.5 \\
\hline
\end{tabular}

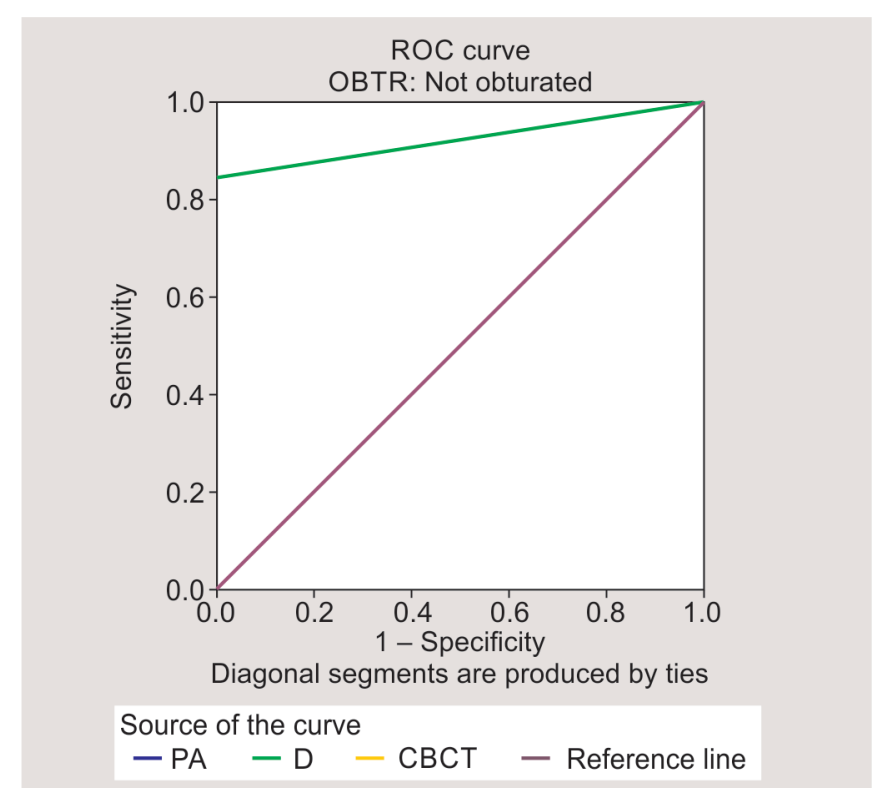

Fig. 2: Receiver operating characteristic curve of accuracy of different radiographic images in detecting vertical root fractures in non-obturated canals

absence of a fracture can only be confirmed by extraction of the tooth in question; which is hard to be consented.

On contrary to studies that have concluded that $C B C T$ imaging is not a reliable method to detect $\mathrm{VRF},{ }^{13-15}$ the present study shows that CBCT appears to be more accurate than conventional dental radiography and digital radiography in the detection of these occurrences. The sensitivity in detecting the VRF in the obturated and non-obturated teeth is highest in the CBCT images. On interpreting the ROC curves, the $\mathrm{CBCT}$ shows accuracy as an almost 


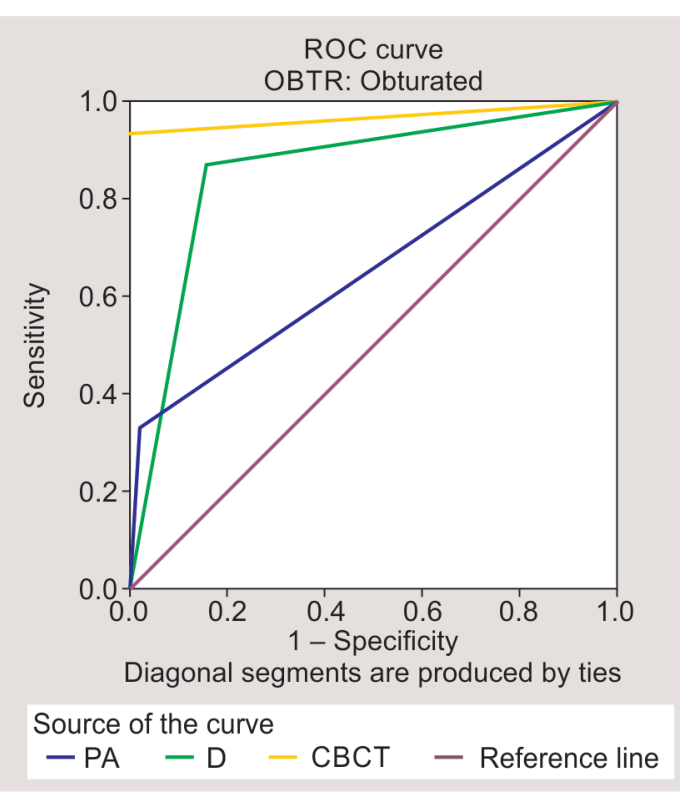

Fig. 3: Receiver operating characteristic curve of accuracy of different radiographic images in detecting vertical root fractures in obturated canals

perfect test in both obturated and non-obturated teeth, whereas the conventional periapical radiograph has poor diagnostic ability in the detection of VRF.

Radiographic images are a 2D representation of a 3D object while CBCT imaging enables the examiner to view the tooth from multiple planes at different angles and different orientations at very thin slices and at a very high contrast, which accounts for higher sensitivity of CBCT in comparison to the periapical radiograph.

In agreement with other studies, ${ }^{16-20}$ the current study showed that the conventional radiographic film has the least sensitivity, specificity, and accuracy compared to the other modalities (digital and $\mathrm{CBCT}$ ) in both obturated and non-obturated groups. For group II (non-root canal treated teeth), none of the observers were able to detect the root fracture with the conventional radiographic film (sensitivity $=0$ ). Better values were observed with the digital images (sensitivity $=86.7 \%$ ). For the specificity (the ability to detect all true negatives) of the imaging modalities, CBCT and digital images produce similar results in the non-root canal treated groups (specificity $=100 \%$ ).

The accuracy of radiographic images and the quality of the images depends on the proper radiographic angulation, contrast, density, and sensitivity of the clinician in interpreting the radiographic findings. ${ }^{21}$ If any component of the imaging chain process is compromised, the resulting image may demonstrate exposure or geometric errors and be suboptimal. ${ }^{22}$ The higher level of accuracy in detecting VRF in the digital images when compared with conventional film for both root canal treated and non-root canal treated groups may be due to better quality images and the ability of changing density and contrast, which can improve the quality of the image. Similar findings were observed in other studies. $7,23,24$

Previous studies reported a decrease in the CBCT specificity when the root canal filling was present because it produces streaking artifacts that might mimic a fracture line. ${ }^{21,25}$ In the current study, the presence of root canal filling did not significantly influence the specificity of CBCT images in detecting VRFs (100\% for both groups). Similar findings were observed in a recent study. ${ }^{26}$ This could be explained by improved resolution of the images produced by the CBCT system used.

Similar to this study, Da Silveira et al. ${ }^{25}$ and Fisekcioglu et al. ${ }^{27}$ reported high specificity, sensitivity, and accuracy of CBCT in detected VRF in teeth with obturated root canals and nonobturated sound teeth. They concluded that the image quality is directly affected by the protocol for achieving the image in the CBCT examination, especially the voxel size. The $0.2 \mathrm{~mm}$ or $0.3 \mathrm{~mm}$ voxel resolution scans were suggested for VRF images. ${ }^{25}$ Other in vivo studies supported the current findings and concluded that CBCT imaging was effective in detecting VRFs with a high sensitivity and specificity (100 and $89.5 \%$ and 80 and $97.5 \%$, respectively). ${ }^{11}$

On the contrary, Hassan et al. ${ }^{9}$ reported variation among the different CBCT systems in their ability to detect VRFs in an ex vivo study and reported more accuracy with the axial slices than the sagittal and coronal views. Similarly, Brady et al. ${ }^{13}$ questioned the benefit of using CBCT for the detection of incomplete VRFs, as the effective dose of the CBCT examinations is significantly higher than that of periapical radiography. Patel et al. ${ }^{14}$ concluded inaccuracy of CBCT in detecting the presence and absence of simulated VRF in root-filled teeth. In their recent systematic review, Chang et al. ${ }^{12}$ could not draw any conclusions regarding diagnostic ability of $\mathrm{CBCT}$ in detecting VRFs.

One reason for variation in results is that CBCT systems vary in their image quality and performance, especially in highly demanding diagnostic tasks such as the detection of VRFs. The superiority of the system in the detection of VRFs can be attributed to the voxel size parameter; ${ }^{26}$ smaller size results in better resolution and contrast. ${ }^{26}$ In the current study, Carestream CS 9000 3D CBCT system at specific exposure parameters was able to produce high-quality images to detect VRFs. Further in vivo researches are required to determine patient scanning and other parameters with $\mathrm{CBCT}$ that could influence the visibility of the fracture line.

\section{Clinical Significance}

It is important to select diagnostic methods that employ low radiation, low cost, and readily available at every clinical setting like the digital radiographic images. If both clinical and the digital radiographic data were not able to provide adequate information, CBCT can be indicated as the imaging method to assess the presence of VRFs.

\section{LiMITATION}

In the present study, the VRFs were mimicked using external forces. The situation in the oral environment may be different as the width of the VRFs in vivo may be less thick than those created in vitro. Further studies on multi-rooted teeth and in vivo conditions are warranted to assess reliability of the imaging modalities.

\section{Conclusion}

With the limitations of this study, it is concluded that CBCT scans are a reliable imaging modality to detect VRFs. It appears to give the highest accuracy in detecting VRFs when compared with the periapical systems in both endodontically and non-endodontically treated teeth. 


\section{References}

1. American Association of Endodontics. Endodontics: Colleagues for Excellence-Cracking the cracked tooth code: Detection and Treatment of Various Longitudinal Tooth Fractures. Chicago: American Association of Endodontics; 2008. pp. 1-8.

2. Bergman $B$, Lundquist $P$, Sjögren U, et al. Restorative and endodontic results after treatment with cast posts and cores. J Prosthet Dent 1989;61(1):10-15. DOI: 10.1016/0022-3913(89)90099-1.

3. Fuss Z, Lustig J, Katz A, et al. An evaluation of endodontically treated vertical root fractured teeth: impact of operative procedures. J Endod 2001;27(1):46-48. DOI: 10.1097/00004770-200101000-00017.

4. Testori T, Badino M, Castagnola M. Vertical root fractures in endodontically treated teeth: a clinical survey of 36 cases. J Endod 1993;19(2):87-91. DOI: 10.1016/S0099-2399(06)81202-1.

5. Chan CP, Lin CP, Tseng SC, et al. Vertical root fracture in endodontically vs nonendodontically treated teeth: a survey of 315 cases in chinese patients. Oral Surg Oral Med Oral Pathol Oral Radio Endod 1999;87(4):504-507. DOI: 10.1016/S1079-2104(99)70252-0.

6. Leubke RG. Vertical crown-root fractures in posterior teeth. Dent Clin North Am 1984;28(4):883-894.

7. Toure B, Faye B, Kane AW, et. al. Analysis of reasons for extraction of endodontically treated teeth: a prospective study. J Endod 2011;37(11):1512-1540. DOI: 10.1016/j.joen.2011.07.002.

8. Moule AJ, Kahler B. Diagnosis and management of teeth with vertical root fractures. Austr Dent J 1999;44(2):75-87. DOI: 10.1111/j.18347819.1999.tb00205.x.

9. Hassan B, Metska ME, Ozok AR, et al. Comparison of five cone beam computed tomography systems for detecting vertical root fractures in endodontically treated teeth. J Endod 2010;36(1):126-129. DOI: 10.1016/j.joen.2009.09.013.

10. Pinsky HM, Dyda S, Pinsky RW, et al. Accuracy of three-dimensional measurements using cone-beam CT. Dentomaxillofacial Radiol 2006;35(6):410-416. DOI: $10.1259 / \mathrm{dmfr} / 20987648$.

11. Talwar S, Utneja S, Nawal RR, et al. Role of cone-beam computed tomography in diagnosis of vertical root fractures: a systematic review and meta-analysis. J Endod 2016;42(1):12-24. DOI: 10.1016/ j.joen.2015.09.012.

12. Chang E, Lam E, Shah P, et al. Cone-beam computed tomography for detecting vertical root fractures in endodontically treated teeth: A systematic review. J Endod 2016;42(2):177-185. DOI: 10.1016/ j.joen.2015.10.005.

13. Brady E, Mannocci F, Brown J, et al. A comparison of CBCT and periapical radiography for the detection of vertical root fractures in non-endodontically treated teeth. Int Endod J 2014;47(8):735-746. DOI: 10.1111/iej.12209.

14. Patel S, Brady E, Wilson R, et al. The detection of vertical root fractures in root filled teeth with periapical radiographs and CBCT scans. Int Endod J 2013;46(12):1140-1152. DOI: 10.1111/iej.12109.
15. Taramsari M, Kajan ZD, Bashirzadeh P, et al. Comparison of highresolution and standard zoom imaging modes in cone beam computed tomography for detection of longitudinal root fracture: an in vitro study. Imaging Sci Dent 2013;43(3):171-177. DOI: 10.5624/ isd.2013.43.3.171.

16. Hassan B, Metska ME, Ozok AR, et al. Detection of vertical root fractures in endodontically treated teeth by a cone beam computed tomography scan. J Endod 2009;355(5):719-722. DOI: 10.1016/ j.joen.2009.01.022.

17. Kajan ZD, Taromsari M. Value of cone beam CT in detection of dental root fractures. Dentomaxillofac Radiol 2012;41(1):3-10. DOI: 10.1259/ dmfr/25194588.

18. Ozer SY. Detection of vertical root fractures of different thicknesses in endodontically enlarged teeth by cone beam computed tomography vs digital radiography. J Endod 2010;36(7):1245-1249. DOI: 10.1016/j. joen.2010.03.021.

19. Bernardes RA, de Moraes IG, Hungaro Duarte MA, et al. Use of conebeam volumetric tomography in the diagnosis of root fractures. Oral Surg Oral Med Oral Pathol Oral Radiol Endod 2009;108(2):270-277. DOI: 10.1016/j.tripleo.2009.01.017.

20. Varshosaz M, Tavakoli MA, Mostafavi M, et al. Comparison of conventional radiography with cone beam computed tomography for detection of vertical root fractures: an in vitro study. J Oral Sci 2010;52(4):593-597. DOI: 10.2334/josnusd.52.593.

21. Wang $P$, Yan XB, Lui DG, et al. Detection of dental root fractures by using cone-beam computed tomography. Dentomaxillofac Radiol 2011;40(5):290-298. DOI: 10.1259/dmfr/84907460.

22. Farman AG. Image guidance: the present future of dental care. Pract Proced Aesthetic Dent 2006;18(6):342-344.

23. Kositbowornchai S, Nuansakul R, Sikram S, et al. Root fracture detection: a comparison of direct digital radiography with conventional radiography. Dentomaxillofac Radiol 2001;30(2): 106-109. DOI: 10.1038/sj.dmfr.4600587.

24. Valizadeh S, Khosaravi M, Azizi Z. Diagnostic accuracy of conventional, digital and cone beam computed tomography in vertical root fracture detection. Iran Endod J 2011;6:15-20.

25. Da Silveira PF, Vizzotto MB, Liedke GS, et al. Detection of vertical root fractures by conventional radiographic examination and cone beam computed tomography-an in vitro analysis. Dent Traumatol 2013;291(1):41-46.

26. Elsaltani MH, Farid MM, Eldin Ashmawy MS. Detection of simulated vertical root fractures: which cone-beam computed tomographic system is the most accurate? J Endod 2016;42(6):972-977. DOI: 10.1016/j.joen.2016.03.013.

27. Fisekcioglu E, Dolekoglu S, Ilguy M, et al. In vitro detection of dental root fractures with cone beam computed tomography (CBCT). Iran J Radiol 2014;11(1):e11485. DOI: 10.5812/iranjradiol. 11485. 